\section{A head start in the cortical space race}

Not all sensory surfaces are created equal. Our sense of touch, for example, is exquisitely good on our hands and face, and less so on our abdomen. How is this so? It has long been recognized that more cortical brain space is devoted to certain sensory surfaces, and the increased brain space probably underlies the heightened perceptual abilities associated with the corresponding areas. It has been estimated, for example, that there is about a hundred times as much cortical tissue devoted to similarly sized regions of the fingers as compared with the abdomen. The same principle of cortical magnification holds true for cortical areas that represent behaviourally critical regions in other species, such as the facial whiskers in rats and the platypus's bill. But how do nerve fibres from certain sensory surfaces capture more cortical space to begin with? By studying a very unusual creature, the star-nosed mole, Kenneth Catania of Vanderbilt University offers some interesting clues as to how this cortical magnification might arise during development.

Star-nosed moles have eleven appendages surrounding each nostril. Each appendage or 'ray' contains thousands of small mechanoreceptor organs, and the moles use their rays to explore the environment and find prey. One ray in particular acts as a

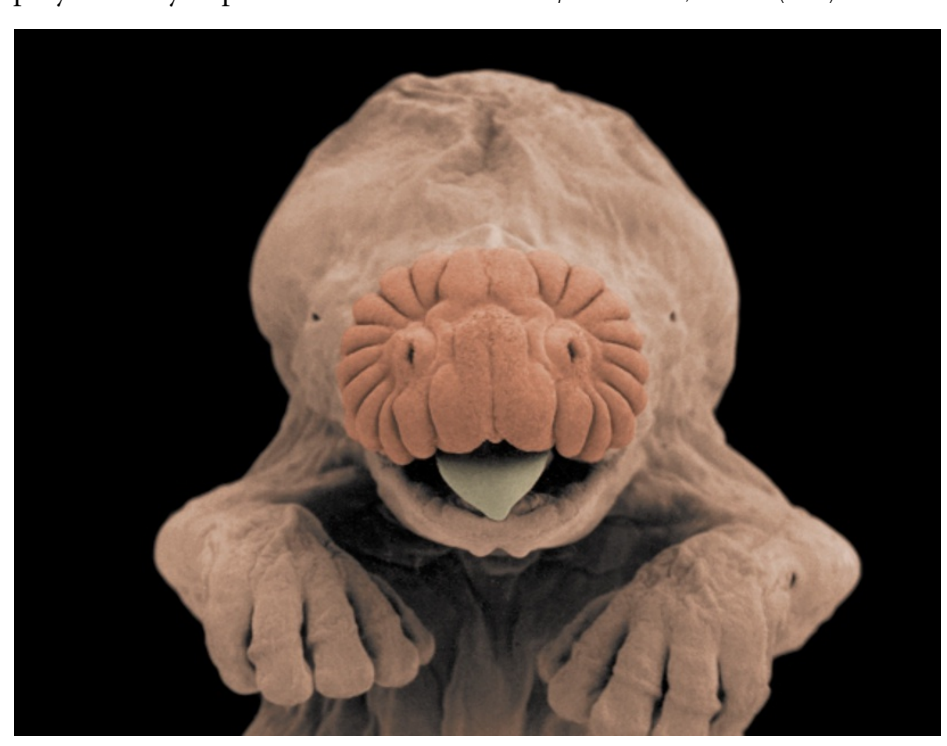

Star-nosed mole embryo. Courtesy of Kenneth C. Catania, Vanderbilt University, Tennessee, USA. not different in any obvious way from the other ten rays. Now, by studying the development of mole embryos, Catania shows that the ray destined to become the fovea has a distinct developmental history: its mechanoreceptor organs mature early and its associated nerve terminals are active in the cortex before the rest. In short, it gets a head start in development, which might give it an early advantage in the competition for cortical space. The results bear striking similarities to observations made in the visual system, and suggest that the final wiring pattern of the brain might be determined in part by the timing of events during development. Starnosed moles might provide a good system to investigate the underlying mechanisms in more detail.

John Spiro, Assistant Editor, Nature Neuroscience

\section{6) References and links} ORIGINAL RESEARCH PAPER Catania, K. C. Early development of a somatosensory fovea: a head start in the cortical space race? Nature Neurosci. 4, 353-354 (2001)

FURTHER READING Catania, K. C. A nose that looks like a hand and acts like an eye: the unusual mechanosensory system of the star-nosed mole. J. Comp. Neurol. 185, 367-372 (1999)

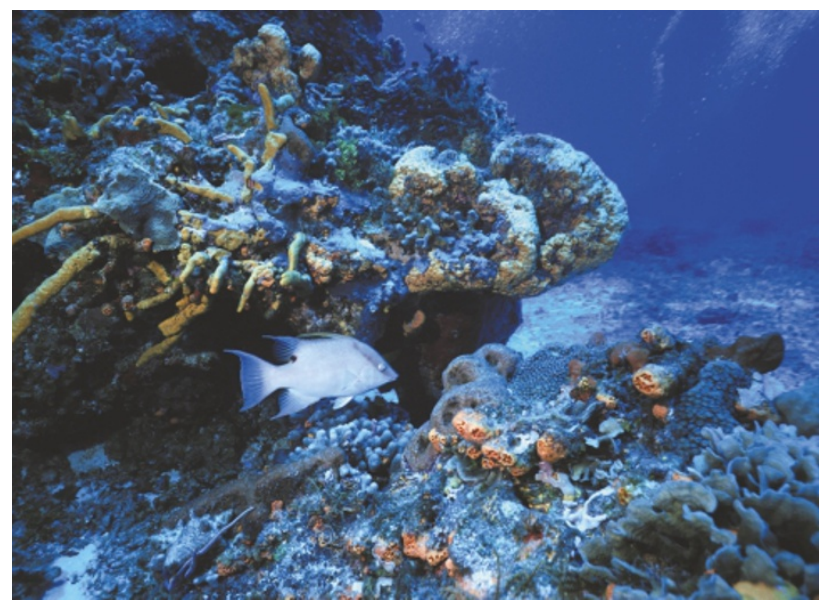

BRAIN EVOLUTION

in the adult cortex, even though it is

\section{Lose the blues}

As anyone who has watched a football match on a black and white television will testify, not seeing things in colour diminishes visual perception, making objects and patterns harder to discern. Colour vision requires at least two types of cone photoreceptor (humans have three) with different spectral sensitivities. Most terrestrial mammals also see in colour, although this is nearly always based on just two types of cone: long-medium-wave-sensitive (L-) cones and short-wave-sensitive ( $\mathrm{S}$-) cones, which have species-dependent maximal sensitivity in the red-green and blue regions, respectively. However, as Peichl et al. now report in the European Journal of Neuroscience, it seems that the S-cones in whales and seals - descendants of terrestrial mammals that returned to the sea - have been lost at some point in their evolution, leaving them with only L-cones and thus essentially colour-blind.

Peichl and colleagues used antibodies specific to either L- or $\mathrm{S}$-opsin, the visual pigment proteins of the respective cone types, to assess the presence of $\mathrm{L}$ - and $\mathrm{S}$-cones in the retinae of seven species of whale and five species of seal, as well as several terrestrial mammals, including close relatives of both whales and seals. All the species studied had a substantial number of L-cones, but in the marine mammals, S-cones were absent. Given the distant relationship between whales and seals, this is indicative of convergent evolution and an adaptive advantage for this trait in the marine enviroment, which is surprising, especially as the spectral composition of light in clear ocean waters is increasingly blue-shifted with depth.

How can this puzzle be explained? As all studied species within each order lack S-opsin, the authors suggest that this loss occurred early in the evolution of both orders, and thus that its adaptive advantage might be related to an early phase in marine mammal evolution rather than to present lifestyles. In coastal sea waters probably inhabited initially by the ancestors of whales and seals in their return to the sea, the underwater light field is red-shifted owing to blue-light absorption by material from land drainage. Consequently, a loss of blue-sensitive S-cones would not be a significant disadvantage. Indeed, the reduction in the complexity of information processing from a monochromatic retina might have freed cortical capacity for other sensory abilities such as echolocation and turbulence tracking. So, it seems that at least for the descendant species that have remained in coastal waters, $\mathrm{S}$-cone loss is probably still useful.

Peter Kirkpatrick

(2) References and links

ORIGINAL RESEARCH PAPER Peichl, L. et al. For whales and seals the ocean is not blue: a visual pigment loss in marine mammals. Eur. J. Neurosci. 13, 1520-1528 (2001) 